\title{
Reference transversely isotropic medium approximating a given generally anisotropic medium
}

\author{
LUDĚK KLIMEŠ
}

Department of Geophysics, Faculty of Mathematics and Physics, Charles University, Ke Karlovu 3, 12116 Praha 2, Czech Republic (http://sw3d.cz/staff/klimes.htm)

Received: February 20, 2017; Revised: August 14, 2017; Accepted: October 16, 2017

\begin{abstract}
For a given stiffness tensor (tensor of elastic moduli) of a generally anisotropic medium, we can estimate the extent to which the medium is transversely isotropic, and determine the direction of its reference symmetry axis. In this paper, we rotate the given stiffness tensor about this reference symmetry axis, and determine the reference transversely isotropic (uniaxial) stiffness tensor as the average of the rotated stiffness tensor over all angles of rotation. The obtained reference transversely isotropic (uniaxial) stiffness tensor represents an analytically differentiable approximation of the given generally anisotropic stiffness tensor. The proposed analytic method is compared with a previous numerical method in two numerical examples.
\end{abstract}

Keywords: elastic anisotropy, stiffness tensor, elastic moduli, transverse isotropy, approximate transverse isotropy, reference symmetry axis, nearest media approximation

\section{INTRODUCTION}

For a given $3 \times 3 \times 3 \times 3$ stiffness tensor (tensor of elastic moduli) of a generally anisotropic medium, we can estimate the extent to which the medium is transversely isotropic, and determine the direction of its reference symmetry axis using the method of Klimeš (2016). The reference symmetry axis of Klimeš (2016) minimizes the Frobenius norm of the angular derivative of the given stiffness tensor about it. The direction of the reference symmetry axis is specified in terms of the unit reference symmetry vector.

If we find that the medium is approximately transversely isotropic (approximately uniaxial), we may wish to determine the transversely isotropic (uniaxial) medium which approximates the given generally anisotropic medium. We shall refer to this transversely isotropic medium as the reference transversely isotropic medium. 


\section{Kliměs}

The stiffness tensor of a transversely isotropic medium is independent of the rotation around the symmetry axis. We thus take the reference symmetry axis determined using the method of Klimeš (2016), rotate the given stiffness tensor about this reference symmetry axis, and determine the reference transversely isotropic stiffness tensor as the average of the rotated stiffness tensor over all angles of rotation. In this way, we obtain an analytically differentiable analytic solution of a problem that was initially solved by brute-force numerical methods (Dellinger, 2005; Norris, 2006; Moakher and Norris, 2006; Kochetov and Slawinski, 2008) or by very rough approximations (Arts et al., 1991). Note that this paper is devoted to the nearest media approximation by a transversely isotropic medium, but not by media of other symmetries.

The lower-case Roman indices take values 1,2 and 3. The Einstein summation over repetitive lower-case Roman indices is used throughout the paper. We consider Cartesian coordinates with unit metric tensor in this paper. In these coordinates, the Frobenius norm of a tensor is independent of the choice of coordinate system orientation.

\section{REFERENCE TRANSVERSELY ISOTROPIC STIFFNESS TENSOR}

We denote the density-reduced stiffness tensor of a given generally anisotropic medium by $a_{i j k l}$. The unit reference symmetry vector $t_{i}$ in the direction of the reference symmetry axis can be obtained using the method of Klimeš (2016).

The projection matrix onto the reference symmetry vector is

$$
Z_{i a}=t_{i} t_{a}
$$

The projection matrix onto the plane perpendicular to the reference symmetry vector is

$$
C_{i a}=\delta_{i a}-t_{i} t_{a},
$$

where the Kronecker delta $\delta_{i n}$ represents the elements of the identity matrix. Minus the generator matrix of the rotation about the reference symmetry vector is

$$
S_{i a}=\varepsilon_{i a r} t_{r}
$$

where $\varepsilon_{i j k}$ is the Levi-Civita symbol.

Then the transformation matrix corresponding to the rotation of vectors about the given reference symmetry vector $t_{i}$ by angle $\varphi$ reads

$$
R_{i a}(\varphi)=Z_{i a}+C_{i a} \cos (\varphi)-S_{i a} \sin (\varphi) .
$$

The rotated stiffness tensor reads

$$
\tilde{a}_{i j k l}(\varphi)=R_{i a}(\varphi) R_{j b}(\varphi) R_{k c}(\varphi) R_{l d}(\varphi) a_{a b c d} \quad,
$$

where $a_{\text {pqrs }}$ without argument $\varphi$ is the given non-rotated tensor.

We define the stiffness tensor $\bar{a}_{i j k l}$ of the reference transversely isotropic medium as the average of the rotated stiffness tensor,

$$
\bar{a}_{i j k l}=\frac{1}{2 \pi} \int_{0}^{2 \pi} \mathrm{d} \varphi \tilde{a}_{i j k l}(\varphi) .
$$




\section{Reference transversely isotropic medium}

On inserting the rotated stiffness tensor (5) with transformation matrix (4) into definition (6), the reference stiffness tensor is composed of terms proportional to the integrals

$$
\begin{gathered}
\frac{1}{2 \pi} \int_{0}^{2 \pi} \mathrm{d} \varphi=1, \\
\frac{1}{2 \pi} \int_{0}^{2 \pi} \mathrm{d} \varphi \cos (\varphi)=0 \\
\frac{1}{2 \pi} \int_{0}^{2 \pi} \mathrm{d} \varphi[\cos (\varphi)]^{2}=\frac{1}{2} \\
\frac{1}{2 \pi} \int_{0}^{2 \pi} \mathrm{d} \varphi[\cos (\varphi)]^{3}=0 \\
\frac{1}{2 \pi} \int_{0}^{2 \pi} \mathrm{d} \varphi \cos (\varphi)[\sin (\varphi)]^{3}=0 \\
\frac{1}{2 \pi} \mathrm{d} \varphi[\cos (\varphi)]^{4}=\frac{3}{8}, \\
\frac{1}{2 \pi} \int_{0}^{2 \pi} \mathrm{d} \varphi \cos \varphi \cos (\varphi) \sin (\varphi)=0 \\
\frac{1}{2 \pi} \int_{0}^{2 \pi} \mathrm{d} \varphi[\cos (\varphi)]_{0}^{2 \pi}[\sin (\varphi)]^{2}=\frac{1}{2 \pi} \int_{0}^{2 \pi} \mathrm{din}(\varphi)=0 \\
\frac{1}{2 \pi} \mathrm{d} \varphi[\sin (\varphi)=0 \\
\int_{0}^{2 \pi} \mathrm{d} \varphi[\sin (\varphi)]^{2}=\frac{1}{2}
\end{gathered}
$$

and

$$
\frac{1}{2 \pi} \int_{0}^{2 \pi} \mathrm{d} \varphi[\sin (\varphi)]^{4}=\frac{3}{8}
$$




\section{Kliměs}

We insert the rotated stiffness tensor (5) with transformation matrix (4) into definition (6), and consider integrals (7)-(21). The stiffness tensor of the reference transversely isotropic medium then reads

$$
\begin{aligned}
\bar{a}_{i j k l}=\{ & Z_{i a} Z_{j b} Z_{k c} Z_{l d} \\
& +\frac{1}{2}\left[Z_{i a} Z_{j b} C_{k c} C_{l d}+Z_{i a} C_{j b} Z_{k c} C_{l d}+Z_{i a} C_{j b} C_{k c} Z_{l d}\right. \\
& \left.+C_{i a} Z_{j b} Z_{k c} C_{l d}+C_{i a} Z_{j b} C_{k c} Z_{l d}+C_{i a} C_{j b} Z_{k c} Z_{l d}\right] \\
+ & \frac{1}{2}\left[Z_{i a} Z_{j b} S_{k c} S_{l d}+Z_{i a} S_{j b} Z_{k c} S_{l d}+Z_{i a} S_{j b} S_{k c} Z_{l d}\right. \\
& \left.+S_{i a} Z_{j b} Z_{k c} S_{l d}+S_{i a} Z_{j b} S_{k c} Z_{l d}+S_{i a} S_{j b} Z_{k c} Z_{l d}\right] \\
+ & \frac{1}{8}\left[C_{i a} C_{j b} S_{k c} S_{l d}+C_{i a} S_{j b} C_{k c} S_{l d}+C_{i a} S_{j b} S_{k c} C_{l d}\right. \\
& \left.+S_{i a} C_{j b} C_{k c} S_{l d}+S_{i a} C_{j b} S_{k c} C_{l d}+S_{i a} S_{j b} C_{k c} C_{l d}\right] \\
+ & \left.\frac{3}{8}\left[C_{i a} C_{j b} C_{k c} C_{l d}+S_{i a} S_{j b} S_{k c} S_{l d}\right]\right\} a_{a b c d} .
\end{aligned}
$$

In the special case of $t_{i}=(0,0,1)$ and $a_{i j k l}=a_{k l i j}$, this equation is equivalent to an analogous equation by Dellinger (2005, Eq. 3).

For any given reference symmetry vector $t_{i}$, the reference transversely isotropic medium (22) minimizes the Frobenius norm of the difference between the given $3 \times 3 \times 3 \times 3$ stiffness tensor $a_{i j k l}$ and the stiffness tensor $\bar{a}_{i j k l}$ of a transversely isotropic medium rotationally invariant with respect to the given reference symmetry vector. However, the reference symmetry vector $t_{i}$ obtained by the analytic method of Klimeš (2016) and recommended in this paper does not minimize the Frobenius norm of the difference exactly; it only approximates the reference symmetry vector of Dellinger (2005), which corresponds to the exact minimum. On the other hand, the reference symmetry vector $t_{i}$ by Klimes (2016) is obtained analytically and is analytically differentiable (Klimeš, 2016; Klimeš and Bulant, 2017).

\section{NUMERICAL EXAMPLES}

We compare the reference transversely isotropic medium determined analytically according to this paper with the reference transversely isotropic medium determined according to Dellinger (2005), who numerically minimizes the Frobenius norm of the difference between the reference transversely isotropic medium and a given stiffness tensor $a_{i j k l}$.

In his first example, Dellinger (2005, Appendix A) considers a transversely isotropic given medium $a_{i j k l}$, calculates the unit symmetry vector, and obtains the reference transversely isotropic medium that coincides with the given medium within rounding errors. Since the given medium in this example is exactly transversely isotropic within rounding errors, the method of Klimeš (2016) yields the unit reference symmetry vector that coincides with the exact symmetry vector within rounding errors, and is thus equal to the unit symmetry vector determined by Dellinger (2005, Appendix A) within rounding errors. The reference transversely isotropic medium (22) then naturally coincides with the given medium within rounding errors. 


\section{Reference transversely isotropic medium}

In his second example, Dellinger (2005, Appendix A) considers a medium $a_{i j k l}$ that is not transversely isotropic. He numerically calculates a unit reference symmetry vector

$$
t_{i}^{\text {exact }}=(0.0525,-0.0056,0.9986)
$$

that minimizes the Frobenius norm of the difference between the given medium and the reference transversely isotropic medium. The approximate analytic method of Klimeš (2016) yields a unit reference symmetry vector

$$
t_{i}=(0.0661,-0.0057,0.9978) \quad .
$$

For the unit reference symmetry vector (23), the reference transversely isotropic medium (22) naturally coincides with the reference transversely isotropic medium determined by Dellinger (2005, Appendix A) within rounding errors. The relative Frobenius norm of the difference between the given medium and the reference transversely isotropic medium corresponding to unit reference symmetry vector (23) is $3.464 \%$. For the unit reference symmetry vector (24) proposed in this paper, the relative Frobenius norm of the difference between the given medium and the reference transversely isotropic medium (22) is $3.482 \%$.

\section{CONCLUSIONS}

The proposed method of determining the reference transversely isotropic stiffness tensor for the given stiffness tensor of a generally anisotropic medium has been coded as a new option of program tiaxis.for of software package FORMS (Bucha and Bulant, 2016).

Gazis et al. (1963) proposed a general but considerably involved method of the nearest media approximation consisting in the normal projection of the given stiffness tensor onto the subset of invariant tensors. It can be proved that the reference transversely isotropic medium (22) obtained here as the average of the rotated stiffness tensor is identical to the transversely isotropic medium obtained by the normal projection of the given stiffness tensor onto the subset of tensors invariant with respect to rotation (5) about a given reference symmetry axis. The reference transversely isotropic medium (22) thus minimizes the Frobenius norm of the difference between given stiffness tensor $a_{i j k l}$ and stiffness tensor $\bar{a}_{i j k l}$ of a transversely isotropic medium for a given reference symmetry axis.

In this paper, we have proposed to determine the reference symmetry axis using the analytic method of Klimeš (2016), instead of numerically minimizing the Frobenius norm of the difference between the given medium and the reference transversely isotropic medium (22) according to Dellinger (2005). The reference symmetry vector obtained by the analytic method of Klimeš (2016) does not minimize the Frobenius norm of the difference exactly, but is obtained analytically and is analytically differentiable (Klimeš and Bulant, 2017).

If the given stiffness tensor of a generally anisotropic viscoelastic medium is not symmetric with respect to the exchange of the first pair of indices and the second pair of indices, the stiffness tensor (22) of the reference medium is rotationally invariant 


\section{Klimeš}

but is not symmetric with respect to the exchange of the first pair of indices and the second pair of indices, and is characterized by three additional parameters in comparison with a transversely isotropic medium (Klime ̌, 2017).

Acknowledgements: The author acknowledges the prior work of Joe Dellinger and his kind suggestions on improving the manuscript. The author has found the numerical examples of Joe Dellinger very useful for this study. The additional suggestions by Alexey Stovas and Petr Jílek made it possible to improve the paper further. The research has been supported by the Grant Agency of the Czech Republic under contract 16-05237S, by the Ministry of Education, Youth and Sports of the Czech Republic within research project CzechGeo/EPOS LM2015079, and by the members of the consortium "Seismic Waves in Complex 3-D Structures" (see "http://sw3d.cz").

\section{References}

Arts R.J., Helbig K. and Rasolofosaon P.N.J., 1991. General anisotropic elastic tensor in rocks: approximation, invariants, and particular directions. SEG Technical Program Expanded Abstracts, 10, 1534-1537.

Bucha V. and Bulant P. (Eds), 2016. SW3D-CD-20 (DVD-ROM). Seismic Waves in Complex 3-D Structures, 26, 183-184 (http://sw3d.cz).

Dellinger J., 2005. Computing the optimal transversely isotropic approximation of a general elastic tensor. Geophysics, 70, I1-I10.

Gazis D.C., Tadjbakhsh I. and Toupin R.A., 1963. The elastic tensor of given symmetry nearest to an anisotropic elastic tensor. Acta Cryst., 16, 917-922.

Klimeš L., 2016. Determination of the reference symmetry axis of a generally anisotropic medium which is approximately transversely isotropic. Stud. Geophys. Geod., 60, 391402.

Klimeš L., 2017. Rotationally invariant viscoelastic medium with a non-symmetric stiffness matrix. Seismic Waves in Complex 3-D Structures, 27, 97-103 (http://sw3d.cz).

Klimeš L. and Bulant P., 2017. Ray tracing and geodesic deviation of the SH and SV reference rays in a heterogeneous generally anisotropic medium which is approximately uniaxial. Stud. Geophys. Geod., 61, 497-512.

Kochetov M. and Slawinski M.A., 2008. On obtaining effective transversely isotropic elasticity tensors. J. Elast., 94, 1-13.

Moakher M. and Norris A.N., 2006. The closest elastic tensor of arbitrary symmetry to an elasticity tensor of lower symmetry. J. Elast., 85, 215-263.

Norris A.N., 2006. Elastic moduli approximation of higher symmetry for the acoustical properties of an anisotropic material. J. Acoust. Soc. Am., 119, 2114-2121. 